\title{
The clinical features of migraine as a manifestation of allergic disease
}

\author{
C. W. M. WILSON \\ M.D., Ph.D., Sc.D., F.R.C.P.E. \\ J. G. KIRKER \\ M.D., F.R.C.P.I. \\ HeCTOR WARNES \\ M.D., Dipl. Psych., M.R.C.Psy., F.R.C.P., F.A.P.A. \\ Moira O'Malley* \\ M.B., Ch.B.

\begin{abstract}
The Departments of Pharmacology, and Psychiatry, University of Dublin, and *St Patrick's Hospital, Dublin, Ireland
\end{abstract}

\begin{abstract}
Summary
Patients with a clinical history of migraine were evaluated psychiatrically, and by electroencephalography. They were challenged with food antigens by skin-prick test, and abdominal symptoms were evaluated following oral ingestion of food allergens. A significant correlation was found between challenge with specific food allergens and the development of migraine headaches, the appearance of abdominal symptoms and the occurrence of positive skin reactions. Psychiatric abnormalities and EEG alterations were associated with the occurrence of headaches and allergic clinical features. It is suggested that the clinical features of migraine can be explained as a result of release of chemical mediators following antigen-antibody reactions in the brain and other tissues where specific antibodies are localized. The continuous ingestion of the responsible food allergens would account for the raised tissue concentrations of noradrenaline, histamine and other mediators to which the clinical features of migraine are attributed.
\end{abstract}

\section{Introduction}

Migraine is a paroxysmal unilateral headache, although it can also be deep-seated and central. It may persist for hours or days, interspersed with periods of complete freedom. The attacks are invariably associated with autonomic disturbances affecting the gastro-intestinal tract-anorexia, nausea, vomiting, constipation or, rarely, diarrhoea, and visual disturbances such as teichopsiae or photophobia (Blau, 1971). Migraine has been classified clinically into the classical type, the ordinary or common migraine, cluster or histamine headaches, and hemiplegic migraine (Critchley, 1970). Attacks may be triggered by numerous factors, but these can be usefully reduced to 6 : food sensitivity, hypoglycaemia, tension, depression, water retention, and menstruation (Dalton, 1973).

Carroll (1971) maintains that migraine is a multifactorial disorder. Each patient should be carefully analysed in order to account for its multiple precipitating and aggravating factors. Any theory concerned with the aetiology of migraine must explain the symptomatology of the condition itself, the function of the vasomotor innervation of the blood vessels which may be affected as the symptoms appear, the mechanisms of action of migraineinducers and trigger-factors, the basis of the beneficial effects of therapeutic agents, and why these agents are more effective in the prodromal phase of the syndrome (Johnson, 1978). The autonomic theory, involving the transient release of noradrenaline from nerve endings to the affected vessels, has been proposed as the most satisfactory theory for explaining the onset of migraine attacks (Johnson, 1978).

The present investigation was designed to identify allergic, familial, psychological and pathophysiological factors related to the onset and occurrence of attacks, in a group of patients diagnosed clinically as suffering from migraine. A psychiatric evaluation of the patients was carried out. Skin and food sensitivity tests were performed in order to identify any allergic basis for their attacks. They were then challenged with specific allergens to which sensitivity had been demonstrated, and investigated by electroencephalography.

\section{Method}

Twenty patients clinically diagnosed as suffering from any of the 4 types of migraine were identified. Their age range was $15-51$ years, mean 34.5 years. Nineteen were female of whom 10 had been married. 
All the patients were in social classes 1 or 2 . The patients filled in a Moos Premenstrual Questionnaire, a Beck Inventory on depression and a HysteroidObsessional Questionnaire (Beck et al., 1961; Caine and Hope, 1967). Skin tests to specific allergens were performed to identify food sensitivities and other extrinsic allergens to which the patients were sensitive (Bock et al., 1978). Food sensitivity analysis was carried out by eliciting the patients' food dislikes and refusal to take specific foods. This is indicative of patients' food sensitivities and confirmed the skin-prick tests (Wilson, 1980). The occurrence of abdominal symptoms including aphthous ulcers, difficulty in swallowing, nausea, abdominal pain, abdominal distension and flatulence, diarrhoea and/or constipation, was correlated with their histories of ingestion of specific foods (Wilson, 1980) and with the development of migraine attacks. Baseline EEGs were performed on all the patients. Ten were then challenged with specific food allergens to which sensitivity had already been established. Repeat EEGs were carried out on 5 of these patients during the resultant migraine attack.

\section{Results}

The characteristics of the migraine attacks are shown in Table 1. Classical migraine was most common. A family history of migraine was obtained from $60 \%$ of the patients. Seventeen described prodromal symptoms in the form of visual or

TABLE 1. Characteristics of migraine and associated symptoms in sample of 20 patients

\begin{tabular}{|c|c|c|c|}
\hline \multicolumn{4}{|c|}{$\begin{array}{c}\text { Age of onset (years): Mean } 34 \cdot 5 \text {, Range } 15-51 \\
\text { Numbers of patients }\end{array}$} \\
\hline \multicolumn{2}{|l|}{ Type } & \multicolumn{2}{|c|}{ Family history } \\
\hline Classical & 15 & Migraine & 15 \\
\hline Common & 3 & Psychiatric & 4 \\
\hline Hemiplegic & 2 & Allergic & \\
\hline Cluster & 0 & & \\
\hline \multirow{3}{*}{\multicolumn{2}{|c|}{ Migraine symptoms }} & Headaches loce & tion \\
\hline & & Left side & \\
\hline & & Right side & \\
\hline Visual disturbances & 11 & Both sides & \\
\hline Taste disturbances & 3 & Deep & \\
\hline Sensitivity to smells & 5 & & \\
\hline Feeling of coldness & 14 & Intensity & \\
\hline Photophobia & 11 & Very severe & 18 \\
\hline Numbness & 4 & Sharp or sickly & \\
\hline Associated & & Frequency & \\
\hline Headache & 20 & $3-120$ per year & \\
\hline Nausea and vomiting & 17 & Mean & \\
\hline Depression & 10 & Predictable & $75 \%$ \\
\hline Fatigue ( $2-7$ days) & 6 & & \\
\hline Slurred speech & 2 & Duration & \\
\hline Rhinitis & 5 & $\begin{array}{l}6 \mathrm{hr} \text { to } 5 \text { days } \\
\text { Average } 2 \text { days }\end{array}$ & \\
\hline
\end{tabular}

sensory disturbances. A feeling of coldness commonly preceded, or was associated with, the migraine attacks, and $20 \%$ described feelings of numbness. Disturbances of taste occurred in 3 patients. These took the form of a craving for sweet or salty foods, which when taken in time, alleviated the attack. The prodromal symptoms were frequently present after the onset of the headache. Apart from headache, the abdominal symptoms of nausea and vomiting were most common in the attacks. Depression occurred in $50 \%$ of the patients and fatigue often persisted for several days. Patients had difficulty in differentiating between depression and fatigue unless specifically questioned. They

TABLE 2. Psychiatric and electroencephalographic evaluation of 20 patients suffering from migraine. Patient numbers

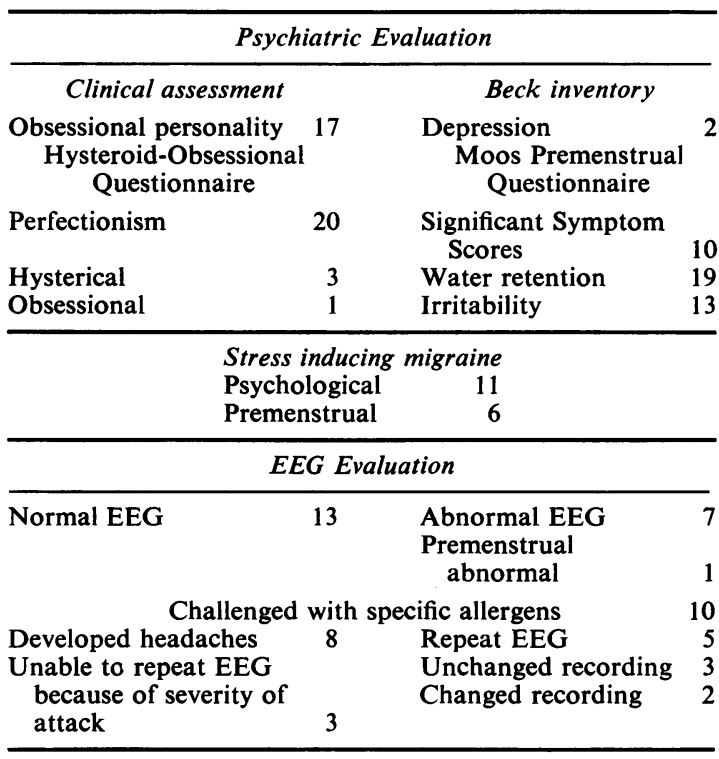

tended to classify these symptoms together as exhausting tiredness, under which 10 of them also described depression. Two with slurred speech had also weakness of one side. These were classified as suffering from hemiplegic migraine.

Psychiatric evaluation of the 20 patients demonstrated the typical self-reported obsessional personality in 17. They had traits of perfectionism, meticulosity, orderliness and high standards of behaviour (Table 2). On the Hysteroid-Obsessional Questionnaire it was noted that the whole group rated high on conscientiousness, stability, fear of change, tension and a desire to live up to high standards and ideals. Three fitted into a more hysterical profile and only one fell clearly into the 
classical obsessional range. In the Beck Inventory, 2 patients scored higher than the rest providing evidence of significant depression. Psychological stress was associated with the occurrence of migraine in 11, and premenstrual tension in 6 of the patients. Resting EEGs were normal in 13 and abnormal in 7 patients. No focal changes were noted in the latter. Ten patients were challenged with particular foods reported in the history as causing migraine attacks, and against which positive skin-prick tests had been obtained. Eight patients developed migraine, of whom 2 showed increased slow wave activity in the EEG.

The hormonal precipitating factor was analysed by the response to the Moos Premenstrual Questionnaire consisting of 8 rating scales containing 44 symptoms. Two rating scales were utilized from this questionnaire: water retention and negative effect. All 19 female patients scored on the water retention scale. Thirteen patients complained of irritability. Ten showed significant symptom scores including the 6 with premenstrual migraine. One of the latter developed an attack during which the EEG also showed a change.

Wine, cheese, chocolate and spirits were the commonest foods precipitating a migraine attack. The foods have been grouped into categories in which there is a common allergen, such as milk protein, which can cause an antigen antibody reaction as shown in the first 2 columns of Table 3 . Specific foods are included in the next 5 columns. Migraine headaches were produced in 12 patients as a result of taking milk protein and 12 patients reacted to the common factor of grapes. Eleven reacted to the specific factor of chocolate. The patients showed positive skin reactions to the classes of foods which caused headaches.

The foods which gave rise to abdominal symptoms were associated with headaches as shown in the first 4 columns. There was a significant correlation between the first 3 types of food sensitivity and the 3 types of clinical response $(P<0.05)$. Tissues giving rise to these clinical responses may therefore be defined as target organs for the allergic reaction.
The abdomen as a target organ is sensitive in a larger number of patients than either skin or brain.

\section{Discussion}

Sensory disturbances were common and very real to the patients in association with their migrainous headaches. The characteristic migrainous headache differed in quality, severity, and frequency between patients. It was accompanied by associated allergic symptoms of which nausea and vomiting, depression and fatigue, and rhinitis were the most common in the present investigation. Johnson (1978) suggests that the different manifestations of migraine are dependent only on the degree of initial vasoconstriction during the prodromal phase, and that prodromal symptoms, and the often accompanying polyuria and frequency, can be explained by an over-release of noradrenaline.

Tension and depression may be triggering factors for the development of migraine (Dalton, 1973), and psychological mechanisms may feature as a cause of migraine attacks (Merskey, 1975).

Obsessional personalities, irritability, and psychological stress were associated with the migraine. Central nervous changes, as shown by abnormalities in EEG recordings, occurred with the attacks following challenge with specific food allergens. The EEG evidence confirms that the headaches are associated with cortical abnormalities. In that the mouth acts as a defence organ through its taste and sensory receptors, it is capable of determining rejection or ingestion of all foreign food proteins. Neurological changes in taste and buccal sensation arise in consequence of the immune reaction. This occurs in the buccal mucosa following ingestion of specific food allergens (Wilson and Sharma, 1979). The close clinical relationship between the psychiatric, neurological and allergic features of the syndrome is indicated by the incidence of psychiatric, allergic and migrainous familial histories among these patients. The incidence of migraine in the general population is approximately $5 \%$. Among epileptics it is $11.1 \%$. A family history of psychiatric illness (Graham, 1967) has been reported in $34 \%$ of

TABLE 3. The effect of antigen challenge on production of migraine headache, reaction to skin-prick test (SPT) following analysis by food sensitivity chart (FSC) and development of abdominal symptoms in 20 patients with a clinical diagnosis of migraine. Numbers of patients having positive reactions to challenge by specific foods

\begin{tabular}{|c|c|c|c|c|c|c|c|c|c|c|c|}
\hline $\begin{array}{l}\text { Antigen } \\
\text { challenges } \\
\text { producing }\end{array}$ & $\begin{array}{c}\text { Cheese } \\
\text { Milk } \\
\text { Cream } \\
\text { Butter }\end{array}$ & $\begin{array}{l}\text { Wine } \\
\text { Fruit } \\
\text { Cake } \\
\text { Grapes }\end{array}$ & Chocolate & Egg & Meat & Tomato & Nut & Fruit & $\begin{array}{l}\text { Cabbage } \\
\text { Lettuce } \\
\text { Spinach }\end{array}$ & $\begin{array}{c}\text { Beans } \\
\text { Peas }\end{array}$ & Cereal \\
\hline Migraine & 12 & 12 & 11 & 3 & 1 & 1 & 1 & 0 & 0 & 0 & 0 \\
\hline Positive SPT & 14 & 13 & 8 & 5 & 5 & 4 & 1 & 9 & 5 & 2 & 5 \\
\hline Positive FSC & 17 & 10 & 17 & 7 & 2 & 2 & 2 & 4 & 4 & 2 & 0 \\
\hline
\end{tabular}


patients. Allergic disorders occur in $35 \%$ of migraine patients and in $38 \%$ of their relatives.

Positive skin-prick tests provide an objective measure of the presence of skin antibodies which react to challenge by intradermal injection of specific food allergens. Food sensitivity analysis by buccal challenge provides a more delicate and discriminating test than skin challenge for detection of specific food allergens (Wilson, 1980). Antibodies are present in the alimentary canal as shown by production of nausea, vomiting, gastric pain and other symptoms which occur as a consequence of challenge with food allergens. The occurrence of migraine was significantly correlated with the production of abdominal symptoms, and the appearance of positive skin-prick tests, on challenge with these allergens. Individuals did not, however, react identically to the same food allergens in the 3 challenge organs. The variable development of migraine headaches, in comparison with the occurence of other symptoms in allergic disease, may be explained in terms of the organ disposition of antibodies, and frequency of ingestion of the specific food allergens.

Noradrenaline, and other catecholamines (Hsu et al., 1977; Johnson, 1978), acetylcholine, 5-hydroxytryptamine (Anthony, Hinterberger and Lance, 1967), histamine, various kinins (Ostfeld, 1960), and Substance $\mathbf{P}$, have all been proposed as mediators in the pathogenesis of migraine. It was suggested by Hanington (1967) that tyramine, and subsequently by Sandler, Youdini and Hanington (1974) that phenylethylamine in the diet, could act as triggering agents in migraine sufferers. In her review on the release of active substances in the lung during anaphylaxis, Piper (1977) provided evidence that all the biologically active mediators proposed as active agents in the pathogenesis of migraine, are released in Type I immune reactions. It has also been suggested that 5-hydroxytryptamine is involved in the anaphylactic reaction (Brocklehurst and Lahiri, 1962).

It is suggested that the basic abnormality in migraine, giving rise to the prodromal and associated symptoms, is an antigen-antibody reaction initiated by a food or other specific allergen. Ingestion of these allergens precipitates the immune reaction in the tissues where specific antibodies are lodged, with subsequent release of chemical mediators. Release of mediators is probably continuously occurring as shown by the continuing low grade presence of abdominal symptoms, taste abnormalities, tiredness and depression, and rhinitis in patients who suffer from allergic disease. When the specific allergen is ingested which also affects the brain, the major shock organ in migraine sufferers, the characteristic headache appears with the associated symptoms.
Any therapeutic agents which inhibit the reaction, or reduce the effects of the mediators at an early stage in the prodromal phase, will be of therapeutic benefit.

Herberg (1975) has suggested that the associated changes in migraine, including somnolence and irritability, might be associated with enhanced lateral hypothalamic activity. However, Johnson (1978) has pointed out that the cause of such postulated abnormal hypothalamic activity is still unknown. There are deficiencies of the blood-brain barrier in the hypothalamus (Wilson and Brodie, 1961; Wilson, Murray and Titus, 1962). It has been demonstrated (Hemmings, 1978a,b) that these deficiencies do allow penetration of food allergens into brain tissue. And so antibody levels may become elevated in the brain tissue as a result of continuing allergen challenge. This could partially account for the high concentrations of chemical mediators in localized areas of the brain subject to allergic challenge, and for the development of migraine and other central symptoms when vasoactive immune concentrations reach a critical level (Editorial, 1974).

\section{References}

ANThONy, M., Hinterberger, H. \& LANCE, J.W. (1967) Plasma serotonin in migraine and stress. Archives of Neurology, 16, 544.

Beck, A.T., Ward, C.H., Mendelson, M., Mock, J. \& ERBAUGH, J. (1961) An inventory for measuring depression. Archives of General Psychiatry, 4, 561.

BlaU, J.N. (1971) Migraine-Research. British Medical Journal, 2, 751.

Bock, S.A., LeE W-Y Remigio, L., Holst, A. \& MAY, C.D. (1978) Appraisal of skin tests with food extracts for diagnosis of food hypersensitivity. Clinical Allergy, 8, 559.

Brocklehurst, W.E. \& LAHIRI, S.C. (1962) The production of bradykinin in anaphylaxis. Journal of Physiology, 160, 15P.

CAINE, T.M. \& Hope, K. (1967) Manual of the HysteroidObsessoid Questionnaire. University of London Press, London.

Carroll, J.D. (1971) Migraine-General management. British Medical Journal, 2, 756.

Critchley, Macdonald (1970) Definition of migraine. In: Third Migraine Symposium. (Ed by Cochrane, A.L.), pp. 181-182. William Heinemann, London.

Dalton, Katharina (1973) Migraine-a personal view. Proceedings of the Royal Society of Medicine, 66, 263.

EDITORIAL (1974) Monoamine oxidase and migraine-a causal link. Lancet, ii, 703.

GrAHAM, J.R. (1967) Cardiac and pulmonary fibrosis during methysergide therapy for headache. American Journal of Medical Sciences, 254, 23.

Hanington, E. (1967) Preliminary report on tyramine headache. British Medical Journal, 1, 550.

Hemmings, W.A. (1967a) The entry into the brain of large molecules derived from dietary protein. Proceedings of the Royal Society (Series B), 200, 175.

HeMmings, W.A. (1978b) Food allergy. Lancet, i, 608.

HerberG, L.J. (1975) The hypothalamus and aminergic pathways in migraine. In: Modern Topics in Migraine. (Ed by Pearce, J.), Chap. 9. Wm Heinemann Medical Books Ltd, London. 
Hsu, L.K.G., Crisp, A.H., KahiRY, R.S., Kaval, J., Chen, C.N., Carruthers, M. \& Zilkha, K. (1977) Early morning migraine. Nocturnal plasma levels of catecholamines, tryptophan, glucose and free fatty acids and sleep encephalographs. Lancet, i, 447.

JoHnson, E.S. (1978) A basis for migraine therapy-the autonomic theory reappraised. Postgraduate Medical Journal, 54, 231.

Merskey, H. (1975) Psychiatric aspects. In: Modern Topics in Migraine. (Ed by Pearce, J.), p. 52. Heinemann Medical Books Ltd, London.

OSTFELD, A.M. (1960) Migraine headache. Its physiology and biochemistry. Journal of the American Medical Association, 174, 1188.

Piper, Priscilla, J. (1977) Anaphylaxis and the release of active substances from the lungs. Pharmacology and Therapeutics (Series 5), 3, 75.
SANDleR, M., Youdini, M.B.H. \& HANington, E. (1974) A phenylethylamine oxidising effect in migraine. Nature. London, 250, 335.

WiLson, C.W.M. (1980) Food sensitivities, taste changes, aphthous ulcers and atopic symptoms in allergic disease. Annals of Allergy, 44, 302.

WILSON, C.W.M. \& BRODIE, B.B. (1961) The absence of the blood-brain barrier from certain areas of the central nervous system. Journal of Pharmacology, 133, 132.

Wilson, C.W.M., Murray, A.W. \& Titus, E. (1962) The effect of reserpine on uptake of epinephrine in brain and certain areas outside the blood-brain barrier. Journal of Pharmacology, 135, 11.

Wilson, C.W.M. \& Sharma, S.C. (1979) An alternative hypothesis for the immune response based on the buccal absorption and action of sodium cromoglycate. In: The Mast Cell. (Ed by Pepys, J. \& Edwards, A.M.), p. 455. Pitman Publishing Co., London. 